\title{
ANÁLISE INTERVALAR E PROJETO DE CONTROLADORES ROBUSTOS VIA PROGRAMAÇÃO ALVO
}

\author{
Alfredo D. S. Lordelo* \\ lordeloddt.fee.unicamp.br
}

\author{
Paulo A. V. Ferreira* \\ valenteddt.fee.unicamp.br
}

${ }^{*}$ Universidade Estadual de Campinas

Faculdade de Engenharia Elétrica e de Computação

Departamento de Telemática

13084-970 Campinas, SP, Brasil

\begin{abstract}
A computational optimization-based approach for designing fixed-order controllers through robust pole placement for uncertain (interval) linear time-invariant SISO plants is presented. The design objective is the minimization of the overall deviation from the desired performance for the closedloop system, as specified by a polytope of characteristic polinomials. The robust pole placement design problem is associated with the solution of an interval Diophantine equation, whose basic properties are analysed. Robust pole placement controllers are viewed as inner solutions of the interval Diophantine equation. Simple and computationally efficient characterizations of the set of all robust pole placement controllers are then obtained and some of its geometric properties discused. Several aspects of the design of robust controllers by Interval Analysis are integrated into a linear goal programming formulation, which can incorporate addicional constraints on the controller parameters. Examples illustrate the main characteristics of the proposed approach.
\end{abstract}

KEYWORDS: Controller design, pole placement, uncertain systems, robust control, goal programming, interval analysis, linear programming.

Artigo submetido em 18/12/02

1a. Revisão em 10/08/04;

Aceito sob recomendação do Ed. Assoc. Prof. Liu Hsu

\section{RESUMO}

É apresentada uma abordagem computacional baseada em otimização para o projeto de controladores de ordem fixa através de alocação robusta de pólos para plantas lineares e invariantes no tempo, SISO, com incertezas do tipo intervalar. O objetivo do projeto é a minimização do desvio total do desempenho desejado para o sistema em malha fechada, especificado por um politopo de polinômios característicos. O problema de projeto por alocação robusta de pólos é associado à solução de uma equação Diofantina intervalar, cujas propriedades básicas são analisadas. Controladores por alocação robusta de pólos são vistos como soluções internas da equação Diofantina intervalar. Caracterizações simples e computacionalmente eficientes do conjunto de todos os controladores por alocação robusta de pólos são então obtidos e algumas das suas propriedades geométricas discutidas. Vários aspectos do projeto de controladores robustos por Análise Intervalar são integrados a uma formulação de programação alvo linear, que também pode incorporar restrições adicionais aos parâmetros do controlador. Exemplos ilustram as principais características da abordagem proposta.

PALAVRAS-CHAVE: Projeto de controladores, alocação de pólos, sistemas incertos, controle robusto, programação alvo, análise intervalar, programação linear. 


\section{INTRODUÇÃO}

O PROBlEMA de projeto de controladores para sistemas lineares e invariantes no tempo, destinados a garantir respostas transiente e de estado estacionário satisfatórias em malha fechada, tem uma solução prática através de alocação de pólos. A técnica é baseada no fato de que muitas especificações de desempenho podem ser satisfeitas utilizando-se realimentação dinâmica da saída para alocar adequadamente os pólos de malha fechada. Uma extensão do problema clássico de alocação de pólos é o problema de alocação regional de pólos, cujo objetivo é alocar os pólos de malha fechada em uma região adequada do plano complexo $s$. O problema de alocação regional de pólos é quase sempre tratado em conexão com o problema mais geral de alocação robusta de pólos, de acordo com o qual, o controlador robusto deve alocar os pólos de malha fechada em uma região específica do plano complexo $s$ face a incertezas com respeito ao modelo matemático da planta. Em muitas situações reais, as incertezas se refletem nos parâmetros da planta, o que tem motivado extensos esforços de pesquisa em Teoria de Controle Robusto Paramétrico (Ackermann, 1993), (Barmish, 1994), (Bhattacharyya et al., 1995).

Neste artigo, plantas incertas são representadas por funções de transferência próprias com coeficientes pertencentes a intervalos reais. O problema de alocação robusta de pólos é formulado como o problema de se alocar robustamente os pólos de malha fechada em uma região especificada através das raízes de um polinômio característico intervalar. Condições semelhantes são adotadas em (Soh et al., 1987), (Rotstein et al., 1991) e (Keel e Bhattacharyya, 1997a). A abordagem proposta para o problema de alocação robusta de polinômios característicos é semelhante às apresentadas em (Soh et al., 1987) e (Keel e Bhattacharyya, 1999). Em (Soh et al., 1987), após se caracterizar explicitamente o conjunto de todos os controladores por alocação robusta de pólos, a distância de um controlador nominal a este conjunto é minimizada utilizando-se uma técnica de Programação NãoLinear. Um método baseado em sensibilidade aplicado a um polinômio característico nominal é utilizado para especificar o polinômio característico intervalar. Em (Rotstein et al., 1991), a distância a um controlador nominal e o tamanho da região de incertezas são relacionados através de uma técnica de projeto por alocação robusta de pólos baseada em Otimização Semi-infinita. Em (Keel e Bhattacharyya, 1997a), alguns desenvolvimentos importantes das últimas duas décadas na área de controle robusto são empregados para tratar o problema de alocação robusta de pólos em uma estrutura de Programação Linear. Em particular, o Teorema das Arestas (Bartlett et al., 1988) é utilizado para obter o conjunto de raízes de polinômios intervalares, permitindo especificações de desempenho para o sistema em malha fechada em termos de critérios comuns no domínio da frequiência como, por exemplo, taxa de amortecimento e grau de estabilidade (Bhattacharyya et al., 1995).

O ponto de partida para todas as técnicas por alocação robusta de pólos mencionadas é a técnica de projeto clássica de alocação de pólos. Como amplamente discutido em (Åström e Wittenmark, 1997) e (Chen, 1999), entre outros, a solução do problema clássico de alocação de pólos pode ser reduzida, sob condições apropriadas, à solução da conhecida equação Diofantina, cuja versão matricial assume a forma de um sistema linear, $\boldsymbol{A x}=\boldsymbol{b}$, na qual $\boldsymbol{A}$ é a matriz de Sylvester associada a uma dada planta de ordem $n, \boldsymbol{x}$ é o vetor com os coeficientes de um controlador de ordem $r$ a ser projetado e $b$ é o vetor com os coeficientes de um dado polinômio característico de grau $n+r$. Dadas $\boldsymbol{A}$ e $\boldsymbol{b}$, existe um controlador $\boldsymbol{x}$, tal que $\boldsymbol{A} \boldsymbol{x}=\boldsymbol{b}$ se e somente se os dois polinômios que descrevem a planta forem coprimos e $r \geq n-1$. Dois polinômios são coprimos se e somente se a resultante de Sylvester associada é não-singular (Chen, 1999).

Este artigo aborda a técnica de projeto de alocação de pólos de maneira sistematizada dentro de conceitos e métodos de Análise Intervalar (Alefeld e Herzberger, 1983), (Moore, 1979). A motivação é levar em conta possíveis representações intervalares, tanto para os coeficientes da planta quanto para os coeficientes do polinômio característico de malha fechada, o que leva à introdução de uma equação Diofantina intervalar, e propor métodos de projeto de controladores baseados nos conceitos de Análise Intervalar. A versão matricial da equação Diofantina intervalar assume a forma de um sistema linear intervalar $[\boldsymbol{A}] \boldsymbol{x}=[\boldsymbol{b}]$, na qual $[\boldsymbol{A}]$ e $[\boldsymbol{b}]$ são a matriz de Sylvester intervalar e o vetor intervalar associados a uma dada planta intervalar e a um dado polinômio característico intervalar, respectivamente. A Análise Intervalar tem se tornado útil para lidar com importantes problemas na área de controle, o que tem se refletido no crescente número de publicações sobre suas aplicações em Sistemas de Controle. Em (Jaulin et al., 2001) é apresentada uma discussão mais detalhada do assunto. A abordagem proposta é apresentada para sistemas com uma entrada e uma saída, mas pode ser estendida para sistemas multi-variáveis (Prado et al., 2004).

Inicialmente, a Seção 2 apresenta a técnica de alocação de pólos e a caracterização da equação Diofantina. As Seções 3 e 4 abordam técnicas clássicas de projeto por alocação de pólos para plantas lineares e invariantes no tempo dentro de uma estrutura de Programação Alvo. As Seções 5 e 6, são dedicadas à caracterização, análise e projeto dos controladores robustos como soluções internas de equações Diofantinas intervalares. Caracterizações simples e computacionalmente eficientes do conjunto de todos os controladores por alocação robusta de pólos são obtidas e algumas das suas propriedades geométricas discutidas. É apresentada uma abordagem com- 
putacional, baseada em otimização, para o projeto de controladores de ordem fixa. Na Seção 7, o uso de Programação Alvo com coeficientes intervalares (Inuiguchi e Kume, 1991) permite uma solução substancialmente mais simples para o problema de alocação robusta do que as apresentadas em (Soh et al., 1987) e (Keel e Bhattacharyya, 1999), e como em (Keel e Bhattacharyya, 1999), todos os problemas de projeto de controladores podem ser reduzidos a problemas de Programação Linear. O objetivo do projeto é a minimização do desvio total do desempenho desejado para o sistema em malha fechada, especificado por um espectro de polinômios característicos. Finalizando, na Seção 8, após caracterizar explicitamente um subconjunto convexo de controladores robustos, o problema de projeto de controladores não-frágeis é formulado como um problema de centralização. Exemplos numéricos ilustram os principais resultados apresentados.

Notação: Dada uma matriz real $\boldsymbol{A}=\left\{a_{i j}\right\}, \boldsymbol{A}^{\boldsymbol{T}}$ denota a transposta de $\boldsymbol{A}$ e $|\boldsymbol{A}|$ é definida como $|\boldsymbol{A}|:=\left\{\left|a_{i j}\right|\right\}$. As matrizes identidade e zero de ordem $n$ são denotadas por $\boldsymbol{I}_{\boldsymbol{n}}$ e $\mathbf{0}_{\boldsymbol{n}}$, respectivamente. Dadas duas matrizes $\boldsymbol{A}$ e $\boldsymbol{B}$ de mesmas dimensões, então $\boldsymbol{A} \leq \boldsymbol{B}$ se e somente se $a_{i j} \leq$ $b_{i j}$ para cada $i, j$. Uma matriz intervalar é definida como $[\boldsymbol{A}]=\left[\boldsymbol{A}^{-}, \boldsymbol{A}^{+}\right]:=\left\{\boldsymbol{A}: \boldsymbol{A}^{-} \leq \boldsymbol{A} \leq \boldsymbol{A}^{+}\right\}$. As matrizes centro e raio de $[\boldsymbol{A}]$ são dadas por $\boldsymbol{A}_{\boldsymbol{c}}:=\left(\boldsymbol{A}^{+}+\boldsymbol{A}^{-}\right) / 2$ e $\boldsymbol{\Delta}:=\left(\boldsymbol{A}^{+}-\boldsymbol{A}^{-}\right) / 2(\boldsymbol{\Delta} \geq \mathbf{0})$, respectivamente. De acordo com esta notação, $A^{-}=A_{c}-\Delta, A^{+}=A_{c}+\Delta$ e $[\boldsymbol{A}]=\left[\boldsymbol{A}_{\boldsymbol{c}}-\boldsymbol{\Delta}, \boldsymbol{A}_{\boldsymbol{c}}+\boldsymbol{\Delta}\right]$. Definições semelhantes se aplicam a um vetor coluna intervalar. Em particular, $[\boldsymbol{b}]=$ $\left[\boldsymbol{b}^{-}, \boldsymbol{b}^{+}\right]=\left[\boldsymbol{b}_{c}-\boldsymbol{\delta}, \boldsymbol{b}_{\boldsymbol{c}}+\boldsymbol{\delta}\right]$.

\section{ALOCAÇÃO DE PÓLOS E A EQUAÇÃO DIOFANTINA}

O problema de projeto de controladores para sistemas lineares e invariantes no tempo, de maneira a garantir respostas transiente e de estado estacionário satisfatórias em malha fechada, tem uma solução prática através de alocação de pólos. A suposição básica é que estabilidade e várias especificações de desempenho podem ser satisfeitas usando-se a realimentação dinâmica da saída para alocar os pólos de malha fechada do sistema em posições apropriadas do plano complexo $s$. Considere o sistema de controle contínuo com realimentação unitária, linear e invariante no tempo, com uma entrada e uma saída (SISO: Single-Input Single-Output), representado na Figura 1.

As funções de transferência de uma dada planta $P(s)$ de ordem $n$, estritamente própria, a ser controlada, e de um controlador série $C(s)$ de ordem $r$, a ser projetado, são representadas por

$$
P(s):=\frac{n_{P}(s)}{d_{P}(s)} \quad \text { e } \quad C(s):=\frac{n_{C}(s)}{d_{C}(s)}
$$

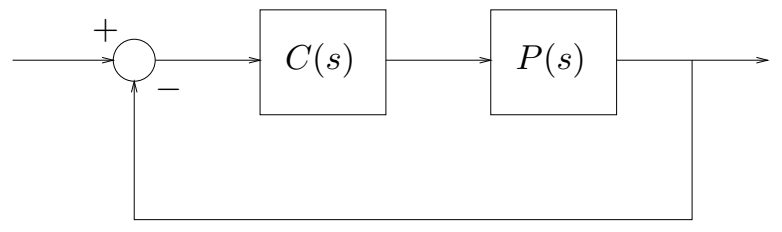

Figura 1: Sistema SISO com realimentação unitária.

nos quais, para $a_{n+2} \neq 0$

$$
\begin{aligned}
n_{P}(s) & :=a_{1} s^{n}+a_{2} s^{n-1}+\ldots+a_{n+1}, \\
d_{P}(s) & :=a_{n+2} s^{n}+a_{n+3} s^{n-1}+\ldots+a_{2 n+2}, \\
n_{C}(s) & :=x_{1} s^{r}+x_{2} s^{r-1}+\ldots+x_{r+1}, \\
d_{C}(s) & :=x_{r+2} s^{r}+x_{r+3} s^{r-1}+\ldots+x_{2 r+2} .
\end{aligned}
$$

Inicialmente, assume-se que os coeficientes da planta $\left(a_{1}, a_{2}, \ldots, a_{2 n+2}\right)$ são precisamente conhecidos. Os parâmetros de projeto do controlador $\left(x_{1}, x_{2}, \ldots, x_{2 r+2}\right)$ devem ser selecionados de maneira que as especificações de desempenho, traduzidas em localizações dos pólos do sistema em malha fechada, sejam satisfeitas com um controlador de menor ordem possível para que o sistema resultante, em malha fechada, tenha um conjunto de $n+r$ pólos desejados. O sistema em malha fechada é representado por

$$
F(s)=\frac{n_{F}(s)}{d_{F}(s)}=\frac{n_{C}(s) n_{P}(s)}{d_{P}(s) d_{C}(s)+n_{P}(s) n_{C}(s)} .
$$

O objetivo desta técnica é alocar os pólos de $F(s)$, ou de forma equivalente, as raízes de $d_{F}(s)$. Para um tratamento completo sobre alocação de pólos, veja por exemplo (Chen, 1999).

O problema de alocação de pólos se resume na solução da equação Diofantina

$$
d_{P}(s) d_{C}(s)+n_{P}(s) n_{C}(s)=d_{F}(s)
$$

para todos os possíveis $d_{F}(s)$, na qual

$$
\begin{aligned}
d_{F}(s) & :=\left(s+p_{1}\right)\left(s+p_{2}\right) \ldots\left(s+p_{n+r}\right), \\
& :=b_{1} s^{n+r}+b_{2} s^{n+r-1}+\ldots+b_{n+r+1} .
\end{aligned}
$$

No entanto, ao invés de resolver a equação (3) diretamente, convém transformá-la num sistema de equações algébricas lineares. Assim, substituindo-se (1) e (4) em (3) e associando os coeficientes com as potências semelhantes em $s$, obtém-se um sistema de $n+r+1$ equações lineares da forma

$$
\begin{aligned}
a_{1} x_{1}+a_{n+2} x_{r+2} & =b_{1}, \\
a_{2} x_{1}+a_{1} x_{2}+\ldots+a_{n+3} x_{r+2}+a_{n+2} x_{r+3} & =b_{2}, \\
\vdots & \\
a_{n+1} x_{r+1}+a_{n+2} x_{2 r+2} & =b_{n+r+1} .
\end{aligned}
$$


Por conveniência, define-se $m:=n+r+1$ e $q:=2 r+2$. Desta forma, (3) pode ser reescrita como uma equação linear da forma

$$
\boldsymbol{A x}=\boldsymbol{b},
$$

na qual

$$
\begin{gathered}
\boldsymbol{A}:=\left[\begin{array}{cccccc}
a_{1} & & & a_{n+2} & & \\
& \ddots & & & \ddots & \\
a_{2} & & a_{1} & a_{n+3} & & a_{n+2} \\
\vdots & \ddots & & \vdots & \ddots & \\
a_{n+1} & & a_{2} & a_{2 n+2} & & a_{n+3} \\
& \ddots & \vdots & & \ddots & \vdots \\
& & a_{n+1} & & & a_{2 n+2}
\end{array}\right] \\
\boldsymbol{x}:=\left[\begin{array}{llll}
x_{1} & x_{2} & \cdots & x_{q}
\end{array}\right]^{T} \in \mathbf{R}^{q}
\end{gathered}
$$

$\mathrm{e}$

$$
\boldsymbol{b}:=\left[\begin{array}{llll}
b_{1} & b_{2} & \cdots & b_{m}
\end{array}\right]^{T} \in \mathbf{R}^{m} .
$$

A matriz $\boldsymbol{A} \in \mathbf{R}^{m \times q}$ em (5) é a matriz de Sylvester associada à planta $P(s)$. Dado que qualquer planta, controlador ou polinômio característico é unicamente especificado por $\boldsymbol{A}, \boldsymbol{x}$ ou $\boldsymbol{b}$, os termos planta $\boldsymbol{A}$, controlador $\boldsymbol{x}$ e polinômio característico $\boldsymbol{b}$, respectivamente, são também utilizados.

Resultados clássicos sobre alocação de pólos mostram que existe uma solução $\boldsymbol{x}$ para a equação Diofantina (3), se e somente se $r \geq n-1$ e $n_{P}(s)$ e $d_{P}(s)$ são coprimos. Se $r<n-1$, então não é possível garantir que (3) terá uma solução para qualquer conjunto desejado de pólos de malha fechada $p_{1}, p_{2}, \ldots, p_{n+r}$ ou, equivalentemente, para qualquer polinômio característico desejado, especificado por $b_{1}, b_{2}, \ldots, b_{n+r+1}$.

\section{ALOCAÇÃO DE PÓLOS VIA PROGRA- MAÇÃO ALVO}

Nesta seção, a técnica de alocação de pólos é analisada no contexto da Programação Alvo (Lordelo e Ferreira, 2002b). A Programação Alvo é uma metodologia tradicional de projeto em diferentes áreas da Engenharia (Ignizio, 1982). O objetivo é obter um controlador de menor ordem possível que aloque um polinômio característico alvo ou aloque robustamente polinômios característicos em uma família politópica de polinômios-alvos. Para um tratamento completo sobre Programação Alvo como técnica independente veja (Yu, 1985).

Uma vez que os alvos podem ou não ser atingidos por um controlador robusto de ordem fixa, uma informação relevante para o projetista seria que alvo é atingível por um controlador de uma determinada ordem. Ajustes são um aspecto importante no processo de decisão baseado no conceito de alvo. Assim, um polinômio característico qualquer $\boldsymbol{b}^{\mathbf{0}}$ é visto como um alvo, que pode ou não ser atingido por um controlador de uma dada ordem $r$.

Considere $\boldsymbol{A} \in \mathbf{R}^{m \times q}$ a matriz de Sylvester associada a uma dada planta $P(s)$. Os vetores

$$
\begin{aligned}
\boldsymbol{\eta} & :=\left[\begin{array}{llll}
\eta_{1} & \eta_{2} & \cdots & \eta_{m}
\end{array}\right]^{T} \\
\boldsymbol{\rho} & :=\left[\begin{array}{llll}
\rho_{1} & \rho_{2} & \cdots & \rho_{m}
\end{array}\right]^{T}
\end{aligned}
$$

e

representam variáveis de desvio em relação ao polinômio característico alvo

$$
\boldsymbol{b}^{\mathbf{0}}:=\left[\begin{array}{llll}
b_{1}^{0} & b_{2}^{0} & \cdots & b_{m}^{0}
\end{array}\right]^{T},
$$

no sentido de que $\boldsymbol{A x}+\boldsymbol{\eta}-\boldsymbol{\rho}=\boldsymbol{b}^{\mathbf{0}}, \operatorname{com} \eta_{i} \geq 0, \rho_{i} \geq 0 \mathrm{e}$ $\eta_{i} \rho_{i}=0$ para todo $i=1,2, \ldots, m$.

A técnica de projeto por alocação de pólos é então reformulada através do seguinte problema de Programação Alvo clássico (Yu, 1985).

\section{Problema 1}

$$
\begin{array}{cc}
\underset{\boldsymbol{x}, \boldsymbol{\eta}, \boldsymbol{\rho}}{\operatorname{minimizar}} & {\left[\sum_{i=1}^{m} \alpha_{i}\left(\eta_{i}+\rho_{i}\right)^{p}\right]^{1 / p}} \\
\text { sujeito a } & \boldsymbol{A} \boldsymbol{x}+\boldsymbol{\eta}-\boldsymbol{\rho}=\boldsymbol{b}^{\mathbf{0}}, \\
& \boldsymbol{\eta} \geq \mathbf{0}, \boldsymbol{\rho} \geq \mathbf{0},
\end{array}
$$

no qual $\alpha_{i}>0, i=1,2, \ldots, m$ são pesos associados aos desvios e $p \geq 1$, usualmente $p=1, p=2$ ou $p=\infty$.

O Problema 1 é sempre factível e é reduzido a um programa linear se $p=1$ ou $p=\infty$, e a um programa quadrático se $p=2$. Como em qualquer formulação de Programação Alvo, o objetivo é minimizar o desvio total de um alvo préestabelecido $\boldsymbol{b}^{\mathbf{0}}$, que por sua vez deve refletir o desempenho desejado para o sistema em malha fechada.

Lema 1 Considere $\left(\boldsymbol{x}^{*}, \boldsymbol{\eta}^{*}, \boldsymbol{\rho}^{*}\right)$ qualquer solução ótima do Problema 1. Então $n_{i}^{*} \rho_{i}^{*}=0$ para todo $i=1,2, \ldots, m$.

A seguir, é estabelecida uma condição necessária e suficiente para a existência de um controlador $\boldsymbol{x}$ de ordem $r$ que aloque o polinômio característico em $\boldsymbol{b}=\boldsymbol{b}^{\mathbf{0}}$, com base no Lema 1 .

Teorema 2 Considere $v_{p}^{*}\left(\boldsymbol{b}^{\mathbf{0}}\right)$ o valor ótimo do Problema 1 e $\boldsymbol{x}^{*}$ o controlador correspondente. Então $\boldsymbol{x}^{*}$ aloca o polinômio característico do sistema de malha fechada em $\boldsymbol{b}=\boldsymbol{b}^{\mathbf{0}}$ se e somente se $v_{p}^{*}\left(\boldsymbol{b}^{\mathbf{0}}\right)=0$. 
Prova: (Yu, 1985) Considere $\left(\boldsymbol{x}^{*}, \boldsymbol{\eta}^{*}, \boldsymbol{\rho}^{*}\right)$ uma solução ótima do Problema 1 e defina $\boldsymbol{b}^{*}:=\boldsymbol{A} \boldsymbol{x}^{*}$. Se $v_{p}^{*}\left(\boldsymbol{b}^{\mathbf{0}}\right)=0$, então $\boldsymbol{\eta}^{*}=\boldsymbol{\rho}^{*}=\mathbf{0}$ e obtém-se $\boldsymbol{b}^{*}=\boldsymbol{b}^{\mathbf{0}}$. Se $v_{p}^{*}\left(\boldsymbol{b}^{\mathbf{0}}\right)>0$, ao menos uma variável de desvio é positiva e pelo Lema 1 , o controlador $\boldsymbol{x}^{*}$ aloca $b_{i}^{*}$ abaixo ou (ou exclusivo) acima de $b_{i}^{0}$, dependendo de qual variável de desvio é positiva.

O Teorema 2 pode ser interpretado em termos da análise clássica de alocação de pólos. Assim, considere $\mathcal{R}(\boldsymbol{A}):=$ $\left\{\boldsymbol{b} \in \mathbf{R}^{m}: \boldsymbol{b}=\boldsymbol{A} \boldsymbol{x}\right\}$ o espaço formado por todos os possíveis polinômios característicos atingíveis por um controlador de ordem $r$. De acordo com o Teorema $2, v_{p}^{*}\left(\boldsymbol{b}^{\mathbf{0}}\right)=0$ se $\boldsymbol{b}^{\mathbf{0}} \in \boldsymbol{\mathcal { R }}(\boldsymbol{A})$, o que sempre acontece quando $n_{P}(s)$ e $d_{P}(s)$ são coprimos e $r \geq n-1$. Se $v_{p}^{*}\left(\boldsymbol{b}^{\mathbf{0}}\right)>0$, então $\boldsymbol{b}^{\mathbf{0}} \notin \mathcal{R}(\boldsymbol{A})$. Em resumo, o controlador $x^{*}$ aloca o polinômio característico em $b^{*}=b^{0}+\eta^{*}-\rho^{*}$, e $b^{*}=b^{0}$ se $b^{0} \in \mathcal{R}(A)$.

Exemplo 1 Considere a função de transferência da planta de segunda ordem apresentada em (Soh et al., 1987)

$$
P(s)=\frac{s+1}{s^{2}-2.2 s-2.4} .
$$

Deseja-se alocar os pólos de malha fechada em $-1 \pm j 0.7 \mathrm{e}$ -10 . O polinômio característico alvo correspondente é

$$
\boldsymbol{b}^{\mathbf{0}}=\left[\begin{array}{llll}
1.000 & 12.00 & 21.49 & 14.90
\end{array}\right]^{T} .
$$

Dado que a solução da equação Diofantina é única quando $r=n-1$, o valor ótimo do Problema 1 é $v_{p}^{*}\left(\boldsymbol{b}^{\mathbf{0}}\right)=0$ para todo $p \geq 1$ e $\boldsymbol{\alpha}>\mathbf{0}$. O alvo $\boldsymbol{b}^{\mathbf{0}}$ é atingido e o controlador de primeira ordem correspondente é

$$
C(s)=\frac{7.688 s+30.53}{s+6.513},
$$

que é o mesmo apresentado em (Soh et al., 1987). É interessante investigar se os pólos dominantes de malha fechada $-1.0 \pm j 0.7$, ou de forma equivalente

$$
\boldsymbol{b}^{\mathbf{0}}=\left[\begin{array}{lll}
1.00 & 2.00 & 1.49
\end{array}\right]^{T},
$$

podem ser alocados usando-se um controlador proporcional, ou seja, $r=0$. Uma análise simples do lugar das raízes mostra que nenhum ganho proporcional é capaz de realizar esta tarefa, conforme ilustra na Figura 2. Os dados numéricos relacionados à solução do Problema 1 para $p=1, p=2 \mathrm{e}$ $p=\infty$ estão resumidos na Tabela 1 .

Tabela 1 - Soluções da Programação Alvo.

\begin{tabular}{cccccc}
\hline \multicolumn{7}{c}{ Ganho } & $\xi$ & $\omega_{n}(\mathrm{rad} / \mathrm{s})$ \\
\hline 1 & $v_{p}$ & $\boldsymbol{b}^{*}$ & 4.05 & 0.72 & 1.22 \\
2 & 0.310 & $(1.000,1.850,1.650)$ & 4.00 & 0.71 & 1.28 \\
$\infty$ & 0.141 & $(1.030,1.848,1.642)$ & 4.00 & 1.20 \\
\hline
\end{tabular}

O fator de amortecimento e a frequiência natural dos pólos em malha fechada desejados $-1.0 \pm j 0.7$ são $\xi=0.82 \mathrm{e}$ $\omega_{n}=1.22 \mathrm{rad} / \mathrm{s}$, respectivamente.

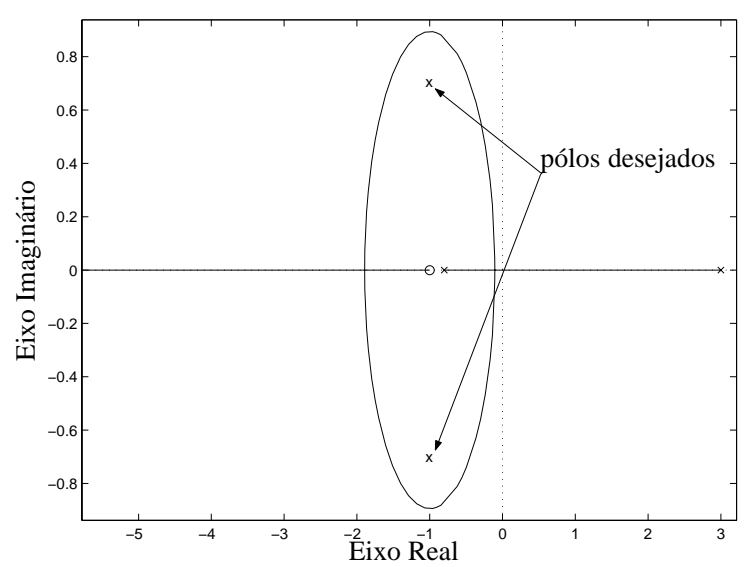

Figura 2: Controle proporcional para o Exemplo 1.

Em princípio, nada pode ser dito sobre o desempenho ou mesmo sobre a estabilidade do sistema em malha fechada quando $v_{p}^{*}\left(\boldsymbol{b}^{\mathbf{0}}\right)>0$. Para associar desempenho e estabilidade às soluções do Problema 1, considere a família de polinômios com coeficientes dentro da bola ponderada pela norma $l_{p}$, de raio $\mu>0$ (Bhattacharyya et al., 1995):

$$
\mathcal{B}_{\boldsymbol{p}}\left(\boldsymbol{b}^{\mathbf{0}}, \mu\right)=\left\{\boldsymbol{b}^{\mathbf{0}}:\left[\sum_{i=1}^{m} \beta_{i}\left|b_{i}-b_{i}^{0}\right|^{p}\right]^{1 / p} \leq \mu\right\},
$$

na qual $\beta_{i}>0, i=1,2, \ldots, m$ são pesos dados e $p \geq 1$. O conjunto $\mathcal{B}_{\boldsymbol{p}}\left(\boldsymbol{b}^{\mathbf{0}}, \mu\right)$ representa uma bola de raio $\mu$ no espaço $l_{p}$ quando $\beta_{1}=\beta_{2}=\cdots=\beta_{m}$. Uma bola $l_{p}$ de polinômios $\mathcal{B}_{\boldsymbol{p}}\left(\boldsymbol{b}^{\mathbf{0}}, \mu\right)$ é Hurwitz se e somente se $\boldsymbol{B}_{\boldsymbol{p}}\left(\boldsymbol{b}^{\mathbf{0}}, \mu\right)$ contém somente polinômios Hurwitz, ou seja, polinômios cujas todas as raízes estiverem no semi-plano esquerdo (aberto) do plano $s$. A margem de estabilidade $l_{p}$ de um dado polinômio $\boldsymbol{b}^{\mathbf{0}}$, definida como o maior raio $\mu$ para o qual $\mathcal{B}_{\boldsymbol{p}}\left(\boldsymbol{b}^{\mathbf{0}}, \mu\right)$ é Hurwitz, pode ser obtida, por exemplo, através do Lugar de Tsypkin-Polyak (Bhattacharyya et al., 1995). Outros procedimentos têm sido propostos na literatura (Barmish, 1994), (Bhattacharyya et al., 1995). Uma conexão entre margens de estabilidade e as soluções ótimas do Problema 1 é estabelecida no Teorema 3.

Teorema 3 Assuma que $\alpha_{i}=\beta_{i}, i=1,2, \ldots, m$, e considere $\mu^{*}$ o raio máximo para o qual $\boldsymbol{B}_{\boldsymbol{p}}\left(\boldsymbol{b}^{\mathbf{0}}, \mu\right)$ é Hurwitz. Se $v_{p}^{*}\left(\boldsymbol{b}^{\mathbf{0}}\right) \leq \mu^{*}$, então $\boldsymbol{b}^{*} \in \mathcal{B}_{p}\left(\boldsymbol{b}^{\mathbf{0}}, \mu^{*}\right)$ e o sistema em malha fechada associado é estável. 
Prova: A prova reside no fato de que $\eta_{i}+\rho_{i}=\left|b_{i}-b_{i}^{0}\right|$, para todo $i=1,2, \ldots, m$, em que $b_{i}$ é a $i$-ésima componente de $\boldsymbol{b}=\boldsymbol{A} \boldsymbol{x}$. Conseqüentemente, $v_{p}^{*}\left(\boldsymbol{b}^{\mathbf{0}}\right)$ é a norma $l_{p}$ mínima entre $\boldsymbol{b}^{\mathbf{0}}$ e $\boldsymbol{\mathcal { R }}(\boldsymbol{A})$. Portanto, se $v_{p}^{*}\left(\boldsymbol{b}^{\mathbf{0}}\right) \leq \mu^{*}$, então $\boldsymbol{b}^{*} \in \mathcal{B}_{\boldsymbol{p}}\left(\boldsymbol{b}^{\mathbf{0}}, \mu^{*}\right)$ e o sistema resultante em malha fechada é estável.

$\mathrm{O}$ aspecto fundamental do Teorema 3 é que $v_{p}^{*}\left(\boldsymbol{b}^{\mathbf{0}}\right)$ é uma medida de proximidade entre $\boldsymbol{b}^{*}$ e $\boldsymbol{b}^{\mathbf{0}}$. Também é sabido que, assumindo-se grau invariante, as raízes de um polinômio variam continuamente em relação aos seus coeficientes (Barmish, 1994). Portanto, para $v_{p}^{*}\left(\boldsymbol{b}^{\mathbf{0}}\right)$ suficientemente pequeno, o sistema em malha fechada associado a $b^{*}$ será estável e o seu desempenho será semelhante ao especificado por $\boldsymbol{b}^{\mathbf{0}}$. Se $v_{p}^{*}\left(\boldsymbol{b}^{\mathbf{0}}\right)$ for grande o suficiente para invalidar o uso do controlador correspondente, uma alternativa é considerar o problema de alocação regional de polinômios característicos. Por simplicidade, todas as formulações de Programação Alvo seguintes serão apresentadas em termos de $p=1 \mathrm{e}$ $\alpha_{i}=1$, para $i=1,2, \ldots, m$.

\section{ALOCAÇÃO EM UM POLITOPO}

Uma extensão do problema de alocação de pólos pontual, em que os pólos de malha fechada são alocados em pontos específicos, é o problema de alocação regional de pólos, cujo objetivo é alocar os pólos de malha fechada em uma região adequada do plano complexo $s$. O problema de alocação regional de pólos é quase sempre tratado em conexão com o problema mais geral de alocação robusta de pólos: o controlador deve alocar os pólos de malha fechada em uma dada região apesar da existência de incertezas com respeito ao modelo matemático da planta. O problema é encontrar $\boldsymbol{b}^{-}$e $\boldsymbol{b}^{+}$ $\left(\boldsymbol{b}^{-} \leq \boldsymbol{b}^{+}\right)$de maneira que todas as raízes da família de polinômios intervalares $[\boldsymbol{b}]:=\left[\boldsymbol{b}^{-}, \boldsymbol{b}^{+}\right]$estejam na região desejada para os pólos de malha fechada. Em (Soh et al., 1987), a determinação de $[\boldsymbol{b}]$ é realizada através de um método baseado em sensibilidade. Outra possibilidade envolve o conceito de conjunto de raízes de uma família politópica. O Teorema das Arestas (Bartlett et al., 1988) estabelece que o conjunto de raízes do hiper-retângulo $[\boldsymbol{b}]$ é limitado pelo conjunto de raízes dos polinômios gerados pelas suas arestas. A idéia de selecionar $[\boldsymbol{b}]$, ou seja, arestas, de maneira a confinar seu conjunto de raízes em uma região adequada do plano complexo $s$ é proposta em (Keel e Bhattacharyya, 1999) e adotada nesta seção.

Uma condição necessária e suficiente para a existência de um controlador de ordem $r$ que aloque um polinômio característico em $[\boldsymbol{b}]$ é estabelecida em termos do problema de Programação Alvo intervalar (Ignizio, 1982):

\section{Problema 2}

$$
\begin{array}{cc}
\text { minimizar } & \sum_{i=1}^{m}\left(\eta_{i}^{-}+\rho_{i}^{+}\right) \\
\boldsymbol{x}, \boldsymbol{\eta}^{-}, \boldsymbol{\rho}^{-}, \boldsymbol{\eta}^{+}, \boldsymbol{\rho}^{+} & \\
\text {sujeito a } & \boldsymbol{A} \boldsymbol{x}+\boldsymbol{\eta}^{-}-\boldsymbol{\rho}^{-}=\boldsymbol{b}^{-}, \\
& \boldsymbol{A} \boldsymbol{x}+\boldsymbol{\eta}^{+}-\boldsymbol{\rho}^{+}=\boldsymbol{b}^{+}, \\
& \boldsymbol{\eta}^{-} \geq \mathbf{0}, \boldsymbol{\rho}^{-} \geq \mathbf{0}, \\
& \boldsymbol{\eta}^{+} \geq \mathbf{0}, \boldsymbol{\rho}^{+} \geq \mathbf{0} .
\end{array}
$$

Teorema 4 Considere $v^{*}([\boldsymbol{b}])$ o valor ótimo do Problema 2, e $\boldsymbol{x}^{*}$ o controlador correspondente. Então $\boldsymbol{x}^{*}$ aloca o polinômio característico do sistema em malha fechada em $[\boldsymbol{b}]:=\left[\boldsymbol{b}^{-}, \boldsymbol{b}^{+}\right]$se e somente se $v^{*}([\boldsymbol{b}])=0$.

Prova: Considere $\left(\boldsymbol{x}^{*}, \boldsymbol{\eta}^{-*}, \boldsymbol{\rho}^{-*}, \boldsymbol{\eta}^{+*}, \boldsymbol{\rho}^{+*}\right)$ uma solução ótima para o Problema 2 e $\boldsymbol{b}^{*}=\boldsymbol{A} \boldsymbol{x}^{*}$. Se $v^{*}([\boldsymbol{b}])=0$, então $\eta^{-*}=\boldsymbol{\rho}^{+*}=\mathbf{0}$ e obtém-se $\boldsymbol{b}^{*} \in[\boldsymbol{b}]$. Se $v^{*}([\boldsymbol{b}])>0$, então ao menos um dos valores $\eta_{i}^{-*}, \rho_{i}^{+*}, i=1,2, \ldots, m$ é positivo e o controlador $\boldsymbol{x}^{*}$ aloca $b_{i}^{*}$ abaixo de $b_{i}^{-}$ou acima de $b_{i}^{+}$, dependendo de qual variável de desvio é positiva.

O Problema 2 exibe as mesmas propriedades básicas do Problema $1(p=1)$ : é um problema de Programação Linear simples e sempre possui uma solução factível. O controlador $\boldsymbol{x}^{*}$ aloca o polinômio característico em $\boldsymbol{b}^{*}=\boldsymbol{b}^{-}-\eta^{-*}+\boldsymbol{\rho}^{-*}$ ou $\boldsymbol{b}^{*}=\boldsymbol{b}^{+}-\boldsymbol{\eta}^{+*}+\boldsymbol{\rho}^{+*}$. Quando $v^{*}([\boldsymbol{b}])>0$, significando que $\boldsymbol{b}^{*} \notin[\boldsymbol{b}]$, o controlador correspondente aloca um polinômio característico com desvio mínimo, no sentido da norma $l_{1}$, em relação ao intervalo $[\boldsymbol{b}]$.

Exemplo 2 Considere a função de transferência da planta de segunda ordem do Exemplo 1 e o problema de alocar os pólos de malha fechada em uma região desejada usando-se um controlador de ordem zero. A região desejada é ilustrada na Figura 3 e corresponde ao espectro de $[\boldsymbol{b}]=\left[\boldsymbol{b}^{-}, \boldsymbol{b}^{+}\right]$definido por

$$
\boldsymbol{b}^{-}=\left[\begin{array}{lll}
1.00 & 1.40 & 1.04
\end{array}\right]^{T}
$$

e

$$
\boldsymbol{b}^{+}=\left[\begin{array}{lll}
1.00 & 2.60 & 1.94
\end{array}\right]^{T} .
$$

A solução ótima do Problema $2, v^{*}([\boldsymbol{b}])=0 \mathrm{e}$

$$
\boldsymbol{b}^{*}=\left[\begin{array}{lll}
1.00 & 1.82 & 1.62
\end{array}\right]^{T},
$$

indica que a alocação em $[\boldsymbol{b}]$ é possível. O controlador $C(s)=4.02$ aloca os pólos de malha fechada em $-0.910 \pm$ $j 0.890$. 


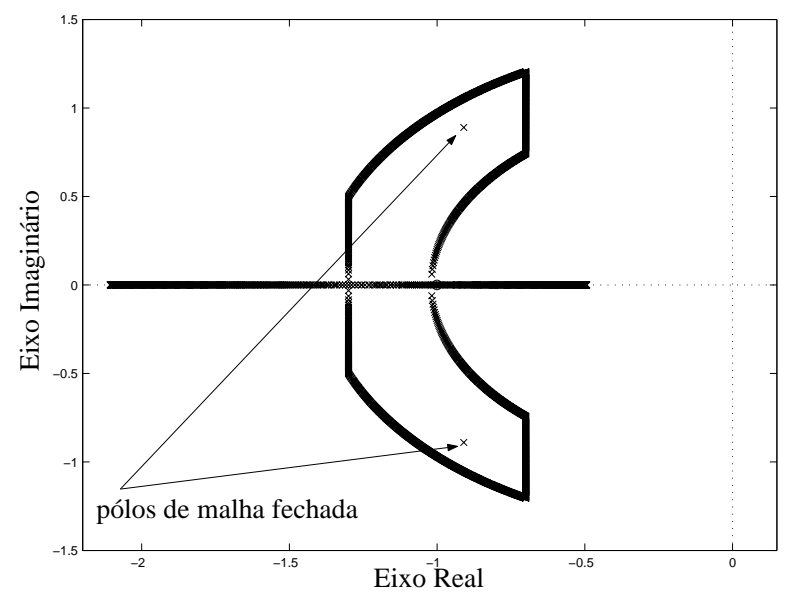

Figura 3: Espectro de $[b(s)]$.

\section{EQUAÇÃO DIOFANTINA INTERVALAR}

Considere o sistema de controle com realimentação unitária linear e invariante no tempo, intervalar, com uma entrada e uma saída (SISO) (Lordelo e Ferreira, 2004). As funções de transferência de uma determinada planta intervalar $[P(s)]$, de ordem $n$, estritamente própria, a ser controlada, e de um controlador série $C(s)$, de ordem $r$, a ser projetado, são representadas como

$$
[P(s)]:=\frac{\left[n_{P}(s)\right]}{\left[d_{P}(s)\right]} \quad \text { e } \quad C(s):=\frac{n_{C}(s)}{d_{C}(s)},
$$

nas quais, $\left[n_{P}(s)\right]$ e $\left[d_{p}(s)\right]$ são polinômios intervalares dados, de maneira que, para $0 \notin\left[a_{n+2}\right]$,

$$
\begin{aligned}
& {\left[n_{P}(s)\right]:=\left[a_{1}\right] s^{n}+\left[a_{2}\right] s^{n-1}+\ldots+\left[a_{n+1}\right]} \\
& {\left[d_{P}(s)\right]:=\left[a_{n+2}\right] s^{n}+\left[a_{n+3}\right] s^{n-1}+\ldots+\left[a_{2 n+2}\right] .}
\end{aligned}
$$

Os polinômios $n_{C}(s)$ e $d_{C}(s)$ estão na forma

$$
\begin{aligned}
n_{C}(s) & :=x_{1} s^{r}+x_{2} s^{r-1}+\ldots+x_{r+1} \\
d_{C}(s) & :=x_{r+2} s^{r}+x_{r+3} s^{r-1}+\ldots+x_{2 r+2}
\end{aligned}
$$

e os parâmetros de projeto do controlador $\left(x_{1}, x_{2}, \ldots, x_{2 r+2}\right)$ devem ser selecionados de maneira que as especificações de desempenho, traduzidas em localizações dos pólos do sistema em malha fechada, sejam satisfeitas com um controlador de menor ordem possível para que o sistema resultante, em malha fechada, tenha um conjunto de $n+r$ pólos desejados.

Os coeficientes incertos da planta intervalar $[P(s)]$ são descritos por intervalos fechados $\left[a_{i}\right]:=\left[a_{i}^{-}, a_{i}^{+}\right]$, para $i=$
$1,2, \ldots, 2 n+2$ e define-se $[\boldsymbol{a}]:=\left[\boldsymbol{a}^{-}, \boldsymbol{a}^{+}\right]$. Note que a descrição de incerteza adotada não assume simetria com respeito aos coeficientes nominais da planta $[P(s)]$. Um coeficiente $\left[a_{i}\right]$ é chamado de propriamente incerto se $a_{i}^{-}<a_{i}^{+}$, para $i=1,2, \ldots, 2 n+2$.

A equação Diofantina intervalar é definida como

$$
\left[d_{P}(s)\right] d_{C}(s)+\left[n_{P}(s)\right] n_{C}(s)=[b(s)],
$$

na qual

$$
[b(s)]:=\left[b_{1}\right] s^{n+r}+\left[b_{2}\right] s^{n+r-1}+\ldots+\left[b_{n+r+1}\right]
$$

é, em geral, um polinômio intervalar que descreve quantidades incertas com respeito ao polinômio característico do sistema em malha fechada. Deve-se notar que $[b(s)]$ é descrito unicamente pelo vetor intervalar $[\boldsymbol{b}]:=\left[\boldsymbol{b}^{-}, \boldsymbol{b}^{+}\right]=$ $\left[\boldsymbol{b}_{c}-\boldsymbol{\delta}, \boldsymbol{b}_{\boldsymbol{c}}+\boldsymbol{\delta}\right]$.

Percebe-se claramente que $\boldsymbol{A} \boldsymbol{x}=\boldsymbol{b}$ pode ser reescrita como uma equação linear intervalar na forma

$$
[\boldsymbol{A}] \boldsymbol{x}=[\boldsymbol{b}]
$$

e, desta forma, incertezas de natureza intervalar associadas aos polinômios intervalares $\left[n_{P}(s)\right]$ e $\left[d_{P}(s)\right]$ levam a uma matriz de Sylvester intervalar $[\boldsymbol{A}]:=\left[\boldsymbol{A}^{-}, \boldsymbol{A}^{+}\right] \in \mathbf{I R}^{m \times q}$, na qual $m:=n+r+1$ e $q:=2 r+2$, cujos limitantes inferior $\boldsymbol{A}^{-}$e superior $\boldsymbol{A}^{+}$são obtidos quando os coeficientes de $[\boldsymbol{A}]$ são substituídos por seus valores inferiores e superiores, respectivamente. Assuma também que a região desejada para os pólos de malha fechada é delimitada por $\mathcal{S}([b(s)])$, o conjunto de raízes do vetor intervalar $[\boldsymbol{b}]$, que especifica o polinômio característico intervalar. Assim,

$$
\begin{aligned}
& {[\boldsymbol{A}]:=\left[\begin{array}{cccccc}
{\left[a_{1}\right]} & & & {\left[a_{n+2}\right]} & & \\
& \ddots & & & \ddots & \\
{\left[a_{2}\right]} & & {\left[a_{1}\right]} & {\left[a_{n+3}\right]} & & {\left[a_{n+2}\right]} \\
\vdots & \ddots & & \vdots & \ddots & \\
{\left[a_{n+1}\right]} & & {\left[a_{2}\right]} & {\left[a_{2 n+2}\right]} & & {\left[a_{n+3}\right]} \\
& \ddots & \vdots & & \ddots & \vdots \\
& & {\left[a_{n+1}\right]} & & & {\left[a_{2 n+2}\right]}
\end{array}\right],} \\
& \boldsymbol{x}:=\left[\begin{array}{llll}
x_{1} & x_{2} & \cdots & x_{q}
\end{array}\right]^{T} \in \mathbf{R}^{q}
\end{aligned}
$$

$\mathrm{e}$

$$
\left.[\boldsymbol{b}]:=\left[\begin{array}{llll}
{\left[b_{1}\right]} & {\left[b_{2}\right]} & \cdots & {\left[b_{m}\right.}
\end{array}\right]\right]^{T} \in \mathbf{I R}^{m} .
$$

O conjunto-solução para equações lineares intervalares na forma $[\boldsymbol{A}] \boldsymbol{x}=[\boldsymbol{b}]$ é definido como

$$
\begin{aligned}
\Sigma:=\{\boldsymbol{x}:[\boldsymbol{A}] \boldsymbol{x}=[\boldsymbol{b}] \text { para alguma } \boldsymbol{A} \in[\boldsymbol{A}] \\
\text { e algum } \boldsymbol{b} \in[\boldsymbol{b}]\} .
\end{aligned}
$$


A seguinte caracterização de $\boldsymbol{\Sigma}$ em termos dos centros e raios de $[\boldsymbol{A}]$ e $[\boldsymbol{b}]$ foi introduzida em (Oettli e Prager, 1964) e (Oettli, 1965).

Teorema 5 (Desigualdade de Oettli-Prager) Considere a equação intervalar $[\boldsymbol{A}] \boldsymbol{x}=[\boldsymbol{b}]$, na qual $[\boldsymbol{A}]=$ $\left[\boldsymbol{A}_{\boldsymbol{c}}-\boldsymbol{\Delta}, \boldsymbol{A}_{\boldsymbol{c}}+\boldsymbol{\Delta}\right] e[\boldsymbol{b}]=\left[\boldsymbol{b}_{\boldsymbol{c}}-\boldsymbol{\delta}, \boldsymbol{b}_{\boldsymbol{c}}+\boldsymbol{\delta}\right]$. Então

$$
\boldsymbol{\Sigma}=\left\{\boldsymbol{x}:\left|\boldsymbol{A}_{\boldsymbol{c}} \boldsymbol{x}-\boldsymbol{b}_{\boldsymbol{c}}\right|-\boldsymbol{\Delta}|\boldsymbol{x}| \leq \boldsymbol{\delta}\right\} .
$$

Prova: Veja (Oettli e Prager, 1964), (Oettli, 1965) e (Rohn, 1989).

O conjunto-solução de $[\boldsymbol{A}] \boldsymbol{x}=[\boldsymbol{b}]$ descreve todos os controladores para os quais existe uma planta $\boldsymbol{A} \in[\boldsymbol{A}]$ e um polinômio característico $\boldsymbol{b} \in[\boldsymbol{b}]$ de maneira que $[\boldsymbol{A}] \boldsymbol{x}=[\boldsymbol{b}]$. A representação de $\boldsymbol{\Sigma}$ em termos dos valores do centro e do raio é descrita no Teorema 5 .

\section{CONTROLADORES ROBUSTOS: SO- LUÇÕES INTERNAS DE $\Sigma$}

Esta seção é dedicada à caracterização e à análise dos controladores robustos como soluções internas de equações Diofantinas intervalares. Considere $[\boldsymbol{A}]$ a matriz de Sylvester intervalar associada a uma planta intervalar $[P(s)]$ e $[\boldsymbol{b}]$ o polinômio característico intervalar especificado.

O conjunto de todas as soluções internas de $[\boldsymbol{A}] \boldsymbol{x}=[\boldsymbol{b}]$ é dado por (Rohn, 1986)

$$
\boldsymbol{\Sigma}_{\mathbf{0}}:=\{\boldsymbol{x}: \boldsymbol{A x} \in[\boldsymbol{b}] \text { para toda } \boldsymbol{A} \in[\boldsymbol{A}]\} .
$$

As propriedades de $\boldsymbol{\Sigma}_{\mathbf{0}}$ são de particular interesse, pois $\boldsymbol{\Sigma}_{\mathbf{0}}$ é precisamente o conjunto de todos os controladores robustos por alocação de pólos. Uma conseqüência importante quando se trata este problema em um contexto de Análise Intervalar é a existência de representações explícitas para $\boldsymbol{\Sigma}_{\mathbf{0}}$. A seguir, são apresentadas caracterizações simples e computacionalmente eficientes dos controladores robustos como soluções internas de equações Diofantinas intervalares.

Teorema 6 (Representações de $\boldsymbol{\Sigma}_{\mathbf{0}}$ ) Considere $\quad \boldsymbol{\Sigma}_{\mathbf{0}} \quad o$ conjunto de todas as soluções internas da equação linear intervalar $[\boldsymbol{A}] \boldsymbol{x}=[\boldsymbol{b}]$, na qual $[\boldsymbol{A}]=\left[\boldsymbol{A}_{\boldsymbol{c}}-\boldsymbol{\Delta}, \boldsymbol{A}_{\boldsymbol{c}}+\boldsymbol{\Delta}\right] \boldsymbol{e}$ $[\boldsymbol{b}]=\left[\boldsymbol{b}_{\boldsymbol{c}}-\boldsymbol{\delta}, \boldsymbol{b}_{\boldsymbol{c}}+\boldsymbol{\delta}\right]$. Defina

$$
\begin{aligned}
& \boldsymbol{\Sigma}_{\mathbf{1}}:=\left\{\boldsymbol{x}:\left|\boldsymbol{A}_{\boldsymbol{c}} \boldsymbol{x}-\boldsymbol{b}_{\boldsymbol{c}}\right|+\boldsymbol{\Delta}|\boldsymbol{x}| \leq \boldsymbol{\delta}\right\} ; \\
& \boldsymbol{\Sigma}_{\mathbf{2}}:=\{\boldsymbol{x}: \boldsymbol{x}=\boldsymbol{y}-\boldsymbol{z}, \\
& A^{-} y-A^{+} z \geq b^{-}, \\
& \boldsymbol{A}^{+} \boldsymbol{y}-\boldsymbol{A}^{-} \boldsymbol{z} \leq \boldsymbol{b}^{+}, \\
& \boldsymbol{y} \geq \mathbf{0}, \boldsymbol{z} \geq \mathbf{0}\} \\
& \boldsymbol{\Sigma}_{\mathbf{3}}:=\left\{(\boldsymbol{x}, \boldsymbol{y}): \boldsymbol{A}_{c} \boldsymbol{x}-\boldsymbol{\Delta} \boldsymbol{y} \geq \boldsymbol{b}^{-},\right. \\
& A_{c} x+\Delta y \leq b^{+}, \\
& -\boldsymbol{y} \leq \boldsymbol{x} \leq \boldsymbol{y}\} \text {. }
\end{aligned}
$$

Então $\boldsymbol{\Sigma}_{\mathbf{0}}=\boldsymbol{\Sigma}_{\mathbf{1}}=\boldsymbol{\Sigma}_{\mathbf{2}}$ e $\boldsymbol{x} \in \boldsymbol{\Sigma}_{\mathbf{0}}$ se e somente se existir um $\boldsymbol{y}$ de maneira que $(\boldsymbol{x}, \boldsymbol{y}) \in \boldsymbol{\Sigma}_{\mathbf{3}}$.

Prova: Veja (Rohn, 1986) para as provas envolvendo as equivalências $\Sigma_{0}=\Sigma_{1}=\Sigma_{2}$. A correspondência entre $\Sigma_{0}$ e $\Sigma_{3}$ é demonstrada em (Kelling, 1994).

A primeira equivalência no Teorema $6, \boldsymbol{\Sigma}_{0}=\boldsymbol{\Sigma}_{1}$, pode ser vista como uma demonstração baseada em Análise Intervalar da condição necessária e suficiente para alocação robusta de pólos, estabelecida em (Soh et al., 1987). Uma diferença sutil é que em (Soh et al., 1987), as notações equivalentes para $\boldsymbol{\Delta}$ e $\boldsymbol{\delta}$ descrevem os desvios de uma planta nominal $\boldsymbol{A}_{\boldsymbol{c}}$ e um polinômio característico nominal $\boldsymbol{b}_{\boldsymbol{c}}$, enquanto que no Teorema $6, \Delta$ e $\delta$ são simplesmente quantidades calculáveis a partir de $\boldsymbol{A}^{-}, \boldsymbol{A}^{+}, \boldsymbol{b}^{-}$e $\boldsymbol{b}^{+}$. De fato, a segunda equivalência do Teorema $6, \boldsymbol{\Sigma}_{\mathbf{0}}=\boldsymbol{\Sigma}_{\mathbf{2}}$, mostra que valores nominais não são necessários para descrever controladores robustos. Note finalmente que a equivalência $\Sigma_{0}=\Sigma_{2}$ e a correspondência entre $\boldsymbol{\Sigma}_{\mathbf{0}}$ e $\boldsymbol{\Sigma}_{\mathbf{3}}$ são especialmente adequadas para manipulações numéricas. Muitas propriedades geométricas úteis relacionadas a $\boldsymbol{\Sigma}_{\mathbf{0}}$ e suas representações equivalentes podem ser analisadas e interpretadas em termos de projeto por alocação robusta de pólos. As proposições seguintes são consequiências imediatas do Teorema 6.

Proposição 1 (Convexidade) $\Sigma_{0}$ é um conjunto convexo poliedral.

A natureza convexa e poliedral de $\boldsymbol{\Sigma}_{\mathbf{0}}$ (como o conjunto de soluções internas) é evidenciada em (Rohn, 1986) através da equivalência $\boldsymbol{\Sigma}_{\mathbf{0}}=\boldsymbol{\Sigma}_{\mathbf{2}}$. A convexidade de $\boldsymbol{\Sigma}_{\mathbf{0}}$ (como o conjunto de controladores robustos) também é citada em (Soh et al., 1987).

Proposição 2 (Existência) $\boldsymbol{\Sigma}_{0}$ é um conjunto não-vazio se e somente se os sistemas de inequações lineares indicados em $\boldsymbol{\Sigma}_{\mathbf{2}}$ ou $\boldsymbol{\Sigma}_{\mathbf{3}}$ têm uma solução. 
A existência de controladores robustos pode ser testada pela Fase I do Algoritmo Simplex quando aplicada às desigualdades lineares em $\boldsymbol{\Sigma}_{\mathbf{2}}$ ou $\boldsymbol{\Sigma}_{\mathbf{3}}$. Quando $\boldsymbol{\Sigma}_{\mathbf{0}}$ é não-vazio, pode-se proceder à seleção de um controlador robusto que satisfaça um dado critério, por exemplo. Deve-se notar que a infactibilidade de $\boldsymbol{\Sigma}_{\mathbf{0}}$ seria causada por uma incompatibilidade entre a descrição intervalar da planta, $[\boldsymbol{A}]=\left[\boldsymbol{A}_{\boldsymbol{c}}-\boldsymbol{\Delta}, \boldsymbol{A}_{\boldsymbol{c}}+\boldsymbol{\Delta}\right]$ e a especificação intervalar do polinômio característico $[\boldsymbol{b}]=$ $\left[\boldsymbol{b}^{-}, \boldsymbol{b}^{+}\right]$. Assumindo que exista um $\boldsymbol{x}$ de maneira que $\boldsymbol{A}_{\boldsymbol{c}} \boldsymbol{x} \in[\boldsymbol{b}]$ e que $\boldsymbol{\Delta}$ é escalonado por um fator não-negativo $\sigma$, a seguinte relação entre a magnitude da incerteza da planta e a existência de controladores robustos pode ser estabelecida.

Proposição 3 (Máxima incerteza) Considere o problema de Programação Não-Linear:

$$
\begin{array}{cc}
\operatorname{maximizar} & \sigma \\
\sigma, \boldsymbol{x}, \boldsymbol{y} & \\
\text { sujeito a } & \boldsymbol{A}_{\boldsymbol{c}} \boldsymbol{x}-(\sigma \boldsymbol{\Delta}) \boldsymbol{y} \geq \boldsymbol{b}^{-}, \\
& \boldsymbol{A}_{\boldsymbol{c}} \boldsymbol{x}+(\sigma \boldsymbol{\Delta}) \boldsymbol{y} \leq \boldsymbol{b}^{+}, \\
& -\boldsymbol{y} \leq \boldsymbol{x} \leq \boldsymbol{y}, \\
& \sigma \geq 0 .
\end{array}
$$

Considere $\sigma^{*}$ o valor ótimo de $\sigma$. Então $\boldsymbol{\Sigma}_{\mathbf{0}}$ é não-vazio se e somente se $\sigma^{*} \geq 1$ e o máximo intervalo de incertezas contendo um controlador robusto é $\left[\boldsymbol{A}_{\boldsymbol{c}}-\sigma^{*} \boldsymbol{\Delta}, \boldsymbol{A}_{\boldsymbol{c}}+\sigma^{*} \boldsymbol{\Delta}\right]$.

Proposição 4 (Limitação) $\boldsymbol{\Sigma}_{\mathbf{0}}$ é limitado se ao menos um coeficiente de $\left[n_{P}(s)\right]$ e um coeficiente de $\left[d_{P}(s)\right]$ são nãodegenerados.

Prova: Note que $\boldsymbol{x} \in \boldsymbol{\Sigma}_{\mathbf{0}}$ implica $\boldsymbol{\Delta}|\boldsymbol{x}| \leq \boldsymbol{\delta}$ (porque $\boldsymbol{x} \in \boldsymbol{\Sigma}_{\mathbf{1}}$ ). Um $\boldsymbol{x}$ ilimitado não pode verificar $\boldsymbol{\Delta}|\boldsymbol{x}| \leq \boldsymbol{\delta}$ se cada coluna de $\boldsymbol{\Delta} \geq \mathbf{0}$ tem ao menos um elemento diferente de zero. Uma vez que $\boldsymbol{\Delta}$ também é uma matriz do tipo Sylvester, conclui-se que $\boldsymbol{\Sigma}_{\mathbf{0}}$ é limitado se ao menos um coeficiente de $\left[n_{P}(s)\right]$ e um coeficiente de $\left[d_{P}(s)\right]$ são nãodegenerados.

Proposição 5 (Limites sobre $\boldsymbol{\Sigma}_{\mathbf{0}}$ ) Suponha que $\boldsymbol{\Sigma}_{\mathbf{0}}$ é um conjunto limitado não-vazio. Para cada $i=1,2, \ldots, q$, defina $x_{i}^{-}$como o valor mínimo do problema linear

$$
\begin{array}{cc}
\underset{\boldsymbol{x}}{\operatorname{minimizar}} & x_{i} \\
\text { sujeito a } & \boldsymbol{x} \in \boldsymbol{\Sigma}_{\mathbf{2}} .
\end{array}
$$

Defina $x_{i}^{+}, i=1,2, \ldots, q$ de maneira semelhante, substituindo minimizar por maximizar. Então o menor vetor intervalar $[\boldsymbol{x}]$ contendo $\boldsymbol{\Sigma}_{\boldsymbol{0}}$ é dado por $[\boldsymbol{x}]:=\left[\boldsymbol{x}^{-}, \boldsymbol{x}^{+}\right]$.
Note que o conhecimento de $[\boldsymbol{x}]$ determina completamente a faixa de variação dos coeficientes de todos os controladores robustos possíveis e constitui uma informação útil para o projeto. De fato, seja $[\boldsymbol{x}]$ particionado da seguinte forma

$$
[\boldsymbol{x}]:=\left[\begin{array}{c}
{\left[\boldsymbol{x}_{\boldsymbol{z}}\right]} \\
\ldots \\
{\left[\boldsymbol{x}_{\boldsymbol{p}}\right]}
\end{array}\right],
$$

na qual $\left[\boldsymbol{x}_{\boldsymbol{z}}\right]$ e $\left[\boldsymbol{x}_{\boldsymbol{p}}\right]$ denotam vetores intervalares de mesma dimensão, $r+1$. Então os zeros e pólos de todos os possíveis controladores por alocação robusta de pólos serão limitados pelo conjunto de raízes dos polinômios intervalares definidos por $\left[\boldsymbol{x}_{\boldsymbol{z}}\right]$ e $\left[\boldsymbol{x}_{\boldsymbol{p}}\right]$, respectivamente. Um exemplo numérico é utilizado para ilustrar este e todos os resultados anteriores relacionados a $\boldsymbol{\Sigma}_{\mathbf{0}}$.

Exemplo 3 Considere a função de transferência da planta de segunda ordem com coeficientes intervalares apresentada em (Soh et al., 1987) e (Rotstein et al., 1991)

$$
[P(s)]=\frac{s+\left[a_{3}\right]}{s^{2}-2.2 s+\left[a_{6}\right]},
$$

na qual $\left[a_{3}\right]=[0.5,1.5]$ e $\left[a_{6}\right]=[-2.6,-2.2]$. Desejase projetar um controlador de primeira ordem $(r=1)$ para alocar de forma robusta os pólos de malha fechada do sistema no conjunto de raízes determinado pelo intervalo $[\boldsymbol{b}]=$ $\left[\boldsymbol{b}^{-}, \boldsymbol{b}^{+}\right]$, com

$$
\boldsymbol{b}^{-}=\left[\begin{array}{llll}
1.000 & 9.600 & 12.81 & 5.120
\end{array}\right]^{T}
$$

$$
\boldsymbol{b}^{+}=\left[\begin{array}{llll}
1.000 & 14.40 & 30.17 & 24.68
\end{array}\right]^{T} .
$$

A matriz de Sylvester intervalar $[\boldsymbol{A}]=\left[\boldsymbol{A}^{-}, \boldsymbol{A}^{+}\right]$é facilmente calculada, assim como $\boldsymbol{A}_{\boldsymbol{c}}, \boldsymbol{\Delta}, \boldsymbol{b}_{\boldsymbol{c}}$ e $\boldsymbol{\delta}$. Existe um controlador por alocação robusta de pólos $\left(\boldsymbol{\Sigma}_{0}\right.$ é não-vazio): a Fase I do método Simplex (Proposição 2) aplicada em $\boldsymbol{\Sigma}_{\mathbf{2}}$ fornece

$$
C_{1}(s)=\frac{13.83 s+6.256}{s-1.852} .
$$

$\mathrm{O}$ espectro do polinômio característico $\left[d_{P}(s)\right] d_{C_{1}}(s)+$ $\left[n_{P}(s)\right] n_{C_{1}}(s)$ (preto), ilustrado na Figura 4, está inteiramente contido no espectro de $[b(s)]$ (cinza).

Uma investigação adicional através da Proposição 3 mostra que $\sigma^{*}=1.319$, implicando que o intervalo máximo de incertezas para a planta pode ser feito cerca de $32 \%$ maior do que o inicialmente especificado. Uma vez que $[P(s)]$ satisfaz as condições da Proposição 4, é possível obter o vetor intervalar $[\boldsymbol{x}]$ utilizando a Proposição 5:

$$
[\boldsymbol{x}]=\left[\boldsymbol{x}^{-}, \boldsymbol{x}^{+}\right]=\left[\begin{array}{c}
{\left[\boldsymbol{x}_{\boldsymbol{z}}\right]} \\
\ldots \\
{\left[\boldsymbol{x}_{\boldsymbol{p}}\right]}
\end{array}\right]=\left[\begin{array}{c}
{\left[\begin{array}{r}
10.13,16.96 \\
-3.979,18.89
\end{array}\right]} \\
\ldots \ldots \ldots \ldots \ldots \ldots \\
{\left[\begin{array}{r}
1.000,1.000 \\
-5.040,1.664
\end{array}\right]}
\end{array}\right] .
$$




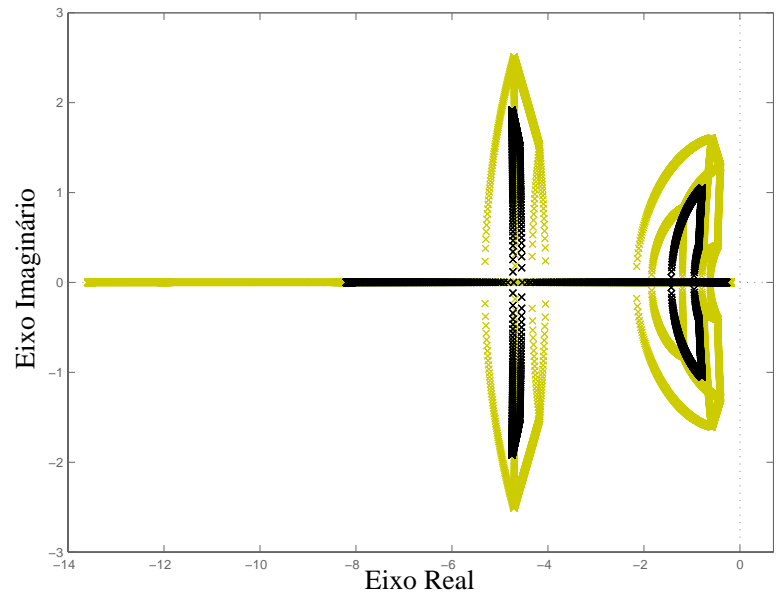

Figura 4: Espectros de $\left[d_{P}(s)\right] d_{C_{1}}(s)+\left[n_{P}(s)\right] n_{C_{1}}(s)$ (preto) e $[b(s)]$ (cinza).

Depois de se calcularem as raízes reais das quatro arestas de $\left[\boldsymbol{x}_{\boldsymbol{z}}\right]$ e da única aresta de $\left[\boldsymbol{x}_{\boldsymbol{p}}\right]$, concluí-se que os zeros e pólos de todos os controladores robustos estão contidos nos intervalos $[-1.865,0.393]$ e $[-1.664,5.040]$, respectivamente. Os pólos de malha fechada podem ser robustamente alocados por controladores estáveis ou instáveis, com zeros de fase mínima ou de fase não-mínima. Neste ponto, não é possível assegurar a existência de um controlador robusto estável e de fase mínima.

\section{ALOCAÇÃO ROBUSTA DE PÓLOS VIA PROGRAMAÇÃO ALVO}

Nesta seção, a existência e o projeto de controladores por alocação robusta de pólos são simultaneamente tratados em uma estrutura de Programação Alvo intervalar com coeficientes intervalares. Convém lembrar que de acordo com o Teorema 6, um vetor $\boldsymbol{x}$ é um elemento do conjunto de controladores robustos $\boldsymbol{\Sigma}_{\mathbf{0}}$ se e somente se $\boldsymbol{x} \in \boldsymbol{\Sigma}_{\mathbf{2}}$.

Teorema 7 De $\boldsymbol{\Sigma}_{\mathbf{2}}$, tem-se que $\boldsymbol{x}=\boldsymbol{y}-\boldsymbol{z}$. Defina $\boldsymbol{\xi}:=$ $\left[\boldsymbol{y}^{T}: \boldsymbol{z}^{T}\right]^{T}$, de maneira que $\boldsymbol{x}=\boldsymbol{T} \boldsymbol{\xi}$, na qual $\boldsymbol{T}:=\left[\boldsymbol{I}_{\boldsymbol{q}} \vdots-\boldsymbol{I}_{\boldsymbol{q}}\right]$, e qualquer especificação adicional $\boldsymbol{x} \in \mathcal{X}$ sobre os coeficientes do controlador pode ser transformada em uma especificação equivalente $\boldsymbol{\xi} \in \boldsymbol{\Xi}$ sobre os componentes de $\boldsymbol{\xi}$. Assim, considere o seguinte problema de Programação Alvo Linear:

$$
\begin{array}{cc}
\underset{\boldsymbol{\xi}, \boldsymbol{\eta}^{-}, \boldsymbol{\rho}^{-}, \boldsymbol{\eta}^{+}, \boldsymbol{\rho}^{+}}{m} & \sum_{i=1}^{m}\left(\eta_{i}^{-}+\rho_{i}^{+}\right) \\
\text {sujeito } a & \\
& \boldsymbol{M}^{-} \boldsymbol{\xi}+\boldsymbol{\eta}^{-}-\boldsymbol{\rho}^{-}=\boldsymbol{b}^{-}, \\
& \boldsymbol{M}^{+} \boldsymbol{\xi}+\boldsymbol{\eta}^{+}-\boldsymbol{\rho}^{+}=\boldsymbol{b}^{+}, \\
& \boldsymbol{\eta}^{-} \geq \mathbf{0}, \boldsymbol{\rho}^{-} \geq \mathbf{0}, \\
& \boldsymbol{\eta}^{+} \geq \mathbf{0}, \boldsymbol{\rho}^{+} \geq \mathbf{0}, \\
\boldsymbol{\xi} \geq \mathbf{0}, \boldsymbol{\xi} \in \mathbf{\Xi},
\end{array}
$$

no qual

$$
M^{-}=\left[\begin{array}{lll}
A^{-} & \vdots & -A^{+}
\end{array}\right], M^{+}=\left[\begin{array}{lll}
A^{+} & \vdots & -A^{-}
\end{array}\right]
$$

$e \boldsymbol{\Xi}$ é um conjunto poliedral não-vazio. Considere $v^{*}$ o valor ótimo deste problema, $\boldsymbol{\xi}^{*}$ o valor ótimo de $\boldsymbol{\xi}$ e $\boldsymbol{x}^{*}=\boldsymbol{T} \boldsymbol{\xi}^{*}$. Então $\boldsymbol{x}^{*} \in \boldsymbol{\Sigma}_{\mathbf{0}} \cap \mathcal{X}$ se e somente se $v^{*}=0$.

Prova: Seja $\left(\boldsymbol{\xi}^{*}, \boldsymbol{\eta}^{-*}, \boldsymbol{\rho}^{-*}, \boldsymbol{\eta}^{+*}, \boldsymbol{\rho}^{+*}\right)$ qualquer solução ótima para o problema do Teorema 7 . Note que otimalidade e a equivalência entre $\boldsymbol{\Xi}$ e $\mathcal{X}$ implicam que $\boldsymbol{x}^{*}=\boldsymbol{T} \boldsymbol{\xi}^{*} \in \mathcal{X}$. Falta mostrar que $\boldsymbol{x}^{*} \in \boldsymbol{\Sigma}_{\mathbf{0}}$ se e somente se $v^{*}=0$.

(Suficiência) Se $v^{*}=0$, então $\boldsymbol{\eta}^{-*}=\boldsymbol{\rho}^{+*}=\mathbf{0}$; portanto

$$
\begin{aligned}
& A^{-} y^{*}-A^{+} z^{*} \geq b^{-} \\
& A^{+} y^{*}-A^{-} z^{*} \leq b^{+} \\
& y^{*} \geq 0, z^{*} \geq 0 .
\end{aligned}
$$

As desigualdades acima possuem uma solução $\left(\boldsymbol{y}^{*}, \boldsymbol{z}^{*}\right)$ e, de acordo com o Teorema $6, \boldsymbol{x}^{*} \in \boldsymbol{\Sigma}_{\mathbf{0}}$. (Necessidade) Qualquer solução ótima para o problema do Teorema 7 é tal que somente um dos $\eta_{i}^{-*}$ e $\rho_{i}^{-*}\left(\eta_{i}^{+*}\right.$ e $\left.\rho_{i}^{+*}\right)$ é positivo (veja (Yu, 1985)). Se $v^{*}>0$, então pelo menos uma componente de $\eta^{-*}$ ou $\rho^{+*}$ é positiva e a componente associada a $\rho^{-*}$ ou $\eta^{+*}$ é nula. Como consequiência, pelo menos uma das desigualdades acima é violada e, novamente pelo Teorema 6 , $\boldsymbol{x}^{*} \notin \boldsymbol{\Sigma}_{\mathbf{0}}$.

O problema do Teorema 7 sempre possui uma solução factível e o seu valor ótimo $v^{*}$ fornece o desvio total de $\boldsymbol{x}^{*} \in \mathcal{X}$ em relação a $\boldsymbol{\Sigma}_{\mathbf{0}}$. Se $v^{*}>0$, então $\boldsymbol{\eta}^{-*}$ e $\boldsymbol{\rho}^{+*}$ indicam quais componentes do alvo intervalar $[\boldsymbol{b}]=\left[\boldsymbol{b}^{-}, \boldsymbol{b}^{+}\right]$não podem ser alcançadas. Quando $v^{*}=0\left(\boldsymbol{\eta}^{-*}=\boldsymbol{\rho}^{+*}=\mathbf{0}\right)$, as variáveis de desvio $\eta^{+*}$ e $\rho^{-*}$ atuam como variáveis de folga e excesso para as desigualdades que descrevem os controladores robustos.

Exemplo 4 Considere a função de transferência da planta incerta de segunda ordem descrita no Exemplo 3. Pode-se verificar que o problema de alocação robusta de polinômios característicos proposto pode ser resolvido por um controlador estável de fase mínima. A restrição $\left[\boldsymbol{I}_{\mathbf{2}} \vdots-\boldsymbol{I}_{\mathbf{2}}\right] \boldsymbol{z} \geq \mathbf{0}$, 
impondo um controlador com coeficientes não-negativos é adicionada ao problema do Teorema 7. O controlador resultante

$$
C_{2}(s)=\frac{11.61 s+14.24}{s+0.428}
$$

é uma solução para o problema de alocação robusta de polinômios, pois $v^{*}=0$. O espectro do polinômio característico $\left[d_{P}(s)\right] d_{C_{2}}(s)+\left[n_{P}(s)\right] n_{C_{2}}(s)$ (preto), ilustrado na Figura 5, está inteiramente contido no espectro de $[b(s)]$ (cinza).

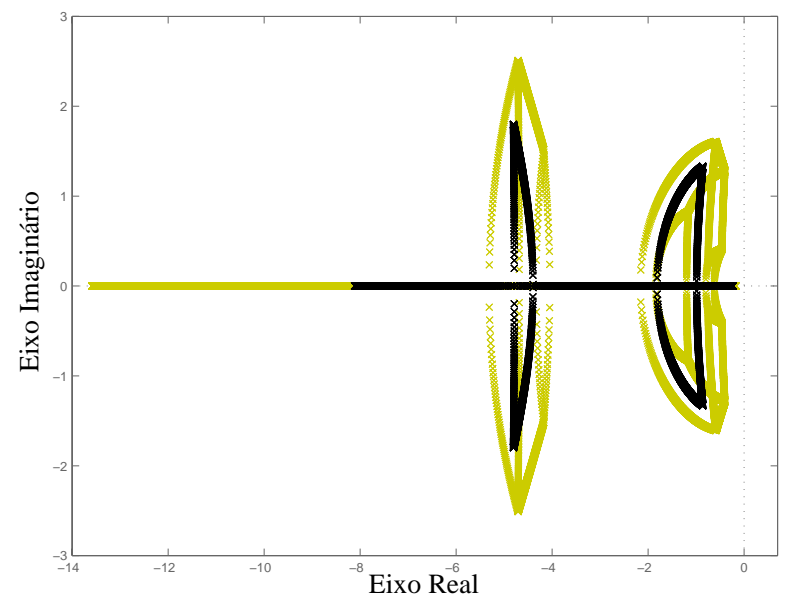

Figura 5: Espectros de $\left[d_{P}(s)\right] d_{C_{2}}(s)+\left[n_{P}(s)\right] n_{C_{2}}(s)$ (preto) e $[b(s)]$ (cinza).

Especificações relacionadas à estrutura dos controladores robustos introduzem restrições adicionais em $\boldsymbol{x}$. Uma especificação popular é a de que o controlador robusto deva apresentar uma estrutura PID na forma paralela

$$
C_{P I D}(s)=\frac{k_{D} s^{2}+k_{P} s+k_{I}}{s},
$$

na qual $k_{D}, k_{P}, k_{I}$ são os ganhos derivativo, proporcional e integral, respectivamente. É comum introduzir limitantes para estes valores: $k_{D} \in\left[k_{D}^{-}, k_{D}^{+}\right], k_{P} \in\left[k_{P}^{-}, k_{P}^{+}\right]$, $k_{I} \in\left[k_{I}^{-}, k_{I}^{+}\right]$. Comparando-se $C_{P I D}(s)$ com $C(s)$ definido em (6) para $r=2$, observa-se que a especificação PID impõe restrições de desigualdade em $x_{1}, x_{2}, x_{3}$ e restrições de igualdade em $x_{4}, x_{5}, x_{6}$. Claramente, $r=2$ é um artifício para a caracterização de um controlador PID e não à sua ordem. A especificação PID correspondente em $\boldsymbol{\xi}$ seria

$$
\boldsymbol{\xi} \in \boldsymbol{\Xi}_{P I D}:=\{\boldsymbol{\xi}: \boldsymbol{G} \boldsymbol{\xi} \leq \boldsymbol{h}, \boldsymbol{E} \boldsymbol{\xi}=\boldsymbol{f}\},
$$

na qual

$$
\boldsymbol{G}:=\left[\begin{array}{ccccccc}
-\boldsymbol{I}_{3} & \vdots & \boldsymbol{0}_{3} & \vdots & \boldsymbol{I}_{3} & \vdots & \boldsymbol{0}_{3} \\
\ldots & \ldots & \ldots & \ldots & \ldots & \ldots \\
\boldsymbol{I}_{3} & \vdots & \boldsymbol{0}_{3} & \vdots & -\boldsymbol{I}_{3} & \vdots & \boldsymbol{0}_{3}
\end{array}\right]
$$

$$
\begin{gathered}
\boldsymbol{E}:=\left[\begin{array}{lllllll}
\mathbf{0}_{3} & \vdots & \boldsymbol{I}_{3} & \vdots & \mathbf{0}_{3} & \vdots & -\boldsymbol{I}_{3}
\end{array}\right], \boldsymbol{f}:=\left[\begin{array}{l}
0 \\
1 \\
0
\end{array}\right], \\
\boldsymbol{h}:=\left[\begin{array}{c}
-\boldsymbol{k}^{-} \\
\ldots \\
\boldsymbol{k}^{+}
\end{array}\right], \boldsymbol{k}^{-}:=\left[\begin{array}{c}
k_{D}^{-} \\
k_{P}^{-} \\
k_{I}^{-}
\end{array}\right], \boldsymbol{k}^{+}:=\left[\begin{array}{c}
k_{D}^{+} \\
k_{P}^{+} \\
k_{I}^{+}
\end{array}\right] .
\end{gathered}
$$

Exemplo 5 O controle PID robusto da planta de terceira ordem

$$
[P(s)]=\frac{\left[a_{3}\right] s+\left[a_{4}\right]}{s^{3}+\left[a_{6}\right] s^{2}+\left[a_{7}\right] s+\left[a_{8}\right]}
$$

é considerado em (Keel e Bhattacharyya, 1997a). Todos os coeficientes incertos são iguais a $[0.99,1.01]$. Deseja-se que os pólos de malha fechada sejam robustamente alocados no conjunto de raízes de $[\boldsymbol{b}]=\left[\boldsymbol{b}^{-}, \boldsymbol{b}^{+}\right]$, com

$$
\boldsymbol{b}^{-}=\left[\begin{array}{lllll}
1.000 & 6.000 & 31.25 & 57.00 & 38.25
\end{array}\right]^{T}
$$

e

$$
\boldsymbol{b}^{+}=\left[\begin{array}{lllll}
1.000 & 14.00 & 45.25 & 77.00 & 54.25
\end{array}\right]^{T} .
$$

Os ganhos PID estão restritos aos intervalos $\left[k_{D}\right] \in[6,14]$, $\left[k_{P}\right] \in[11,19]$ e $\left[k_{I}\right] \in[31,39]$. Para respeitar as condições $r=2$ e $x_{4}=0$, as componentes de $\boldsymbol{b}^{-}$e $\boldsymbol{b}^{+}$são deslocadas para a direita e zeros são incluídos nas primeiras posições de vetores aumentados $\boldsymbol{b}^{-}$e $\boldsymbol{b}^{+}$. Então fazendo $\boldsymbol{\Xi}=\boldsymbol{\Xi}_{P I D}$, a solução ótima do problema do Teorema 7 fornece $v^{*}=0$, e o controlador PID robusto correspondente

$$
C_{P I D_{1}}(s)=\frac{12.43 s^{2}+18.76 s+38.87}{s} .
$$

A abordagem por Análise Intervalar mostra que somente dois vértices de $\boldsymbol{A}$ são necessários para formular problemas de alocação robusta de pólos. O número de desigualdades lineares na formulação por Programação Alvo é $2 n+2 r+2$ e aumenta linearmente com $n$ e $r$. Na abordagem por Programação Linear proposta em (Keel e Bhattacharyya, 1997a), o número de desigualdades lineares, que resulta de uma operação combinatorial com todos os vértices de $\boldsymbol{A}$, aumenta exponencialmente com $n$ e $r$. O controlador PID encontrado por este método foi

$$
C_{P I D_{2}}(s)=\frac{12.5 s^{2}+17.75 s+38.25}{s} .
$$

\section{NÃO-FRAGILIDADE DE CONTROLADO- RES ROBUSTOS}

Um objetivo importante no projeto de controladores em sistemas realimentados é evitar projetos frágeis, nos quais pequenas variações nos coeficientes do controlador projetado podem deteriorar o desempenho do sistema em malha fechada 
de forma significativa (Keel e Bhattacharyya, 1997b). Nesta seção é proposto um procedimento para o projeto de controladores baseado na solução de um problema de centralização, de maneira a obter o máximo desvio nos coeficientes do controlador a partir dos seus valores nominais. Problemas de centralização clássicos na área de Otimização Global são apresentados em (Horst e Pardalos, 1994). No presente contexto, a idéia é determinar um controlador central $\boldsymbol{x}$ e o máximo raio $\theta$, tais que

$$
\boldsymbol{x}+\theta \mathcal{C} \in \boldsymbol{\Sigma}_{\mathbf{0}},
$$

sendo $\mathcal{C}$ um determinado conjunto convexo que descreve como os coeficientes do controlador podem variar e $\boldsymbol{x}+$ $\theta \mathcal{C}:=\left\{\boldsymbol{x}+\theta \boldsymbol{v}, \boldsymbol{v} \in \boldsymbol{\Sigma}_{\mathbf{0}}\right\}$.

$\mathrm{O}$ raio $\theta$ representa uma medida da não-fragilidade do controlador robusto $\boldsymbol{x}$ quando os seus coeficientes variam de acordo $\operatorname{com} \mathcal{C}$. Assumindo que $\mathcal{C}$ é um hiper-retângulo, ou seja, um vetor intervalar em $\mathbf{I R}^{q}$, o problema de projeto do controlador assume a seguinte forma:

\section{Problema 3}

$$
\begin{array}{cc}
\underset{\theta, \boldsymbol{x}, \boldsymbol{y}}{\operatorname{maximizar}} & \theta \\
\text { sujeito a } & \boldsymbol{A}_{\boldsymbol{c}}\left(\boldsymbol{I}_{\boldsymbol{q}}+\theta \boldsymbol{T}\right) \boldsymbol{x}-\boldsymbol{\Delta} \boldsymbol{y} \geq \boldsymbol{b}^{-}, \\
& \boldsymbol{A}_{\boldsymbol{c}}\left(\boldsymbol{I}_{\boldsymbol{q}}-\theta \boldsymbol{T}\right) \boldsymbol{x}-\boldsymbol{\Delta} \boldsymbol{y} \geq \boldsymbol{b}^{-}, \\
& \boldsymbol{A}_{\boldsymbol{c}}\left(\boldsymbol{I}_{\boldsymbol{q}}+\theta \boldsymbol{T}\right) \boldsymbol{x}+\boldsymbol{\Delta} \boldsymbol{y} \leq \boldsymbol{b}^{+}, \\
& \boldsymbol{A}_{\boldsymbol{c}}\left(\boldsymbol{I}_{\boldsymbol{q}}-\theta \boldsymbol{T}\right) \boldsymbol{x}+\boldsymbol{\Delta} \leq \boldsymbol{b}^{+}, \\
& -\boldsymbol{y} \leq\left(\boldsymbol{I}_{\boldsymbol{q}}+\theta \boldsymbol{T}\right) \boldsymbol{x} \leq \boldsymbol{y}, \\
& -\boldsymbol{y} \leq\left(\boldsymbol{I}_{\boldsymbol{q}}-\theta \boldsymbol{T}\right) \boldsymbol{x} \leq \boldsymbol{y}, \\
& \theta \geq 0,
\end{array}
$$

na qual, $\boldsymbol{T}:=\operatorname{diag}\left(t_{1}, t_{2}, \ldots, t_{q}\right)$, com $t_{i}=1$, se a variação no $i$-ésimo componente de $\boldsymbol{x}$ é considerada, e $t_{i}=0$, caso contrário.

\section{CONCLUSÕES}

A conclusão mais importante deste artigo é que a análise e o projeto de controladores por alocação robusta de pólos podem ser sistematizados através de Análise Intervalar. A caracterização do conjunto dos controladores robustos como soluções internas de uma equação Diofantina intervalar criou condições para se obter muitas propriedades úteis relacionadas ao problema de alocação robusta de pólos. Como consequiência da utilização dos conceitos de Análise Intervalar e suas interpretações em termos de alocação robusta de pólos, o projeto de controladores robustos foi realizado através de uma abordagem por Programação Alvo Linear, que combina a facilidade de implementação e eficiência dos algoritmos de Otimização Linear com uma representação linear adequada dos aspectos de tomada de decisão envolvidos. Em particular, a utilização dos resultados de Análise
Intervalar permitiu uma solução mais simples para o problema de alocação robusta de pólos, quando comparada com as metodologias apresentadas em (Soh et al., 1987) e (Keel e Bhattacharyya, 1999). A caracterização de um sub-conjunto convexo de controladores robustos permitiu a formulação do problema de projeto de controladores não-frágeis como um problema de centralização. Os procedimentos de projeto propostos são facilmente implementados com base em programas computacionais existentes.

\section{AGRADECIMENTOS}

Este trabalho foi financiado pelo Conselho Nacional de Desenvolvimento Científico e Tecnológico - CNPq.

\section{REFERÊNCIAS}

Ackermann, J. (1993). Robust Control: Systems with Uncertain Physical Parameters, Springer-Verlag, New York, $N Y$.

Alefeld, G. e Herzberger, J. (1983). Introduction to Interval Computations, Academic Press, New York, NY.

Åström, K. J. e Wittenmark, B. (1997). Computer-Controlled Systems: Theory and Design, 3rd. edition, PrenticeHall, Upper Saddle River, NJ.

Barmish, B. R. (1994). New Tools for Robustness of Linear Systems, Macmillan Publishing Co., New York, NY.

Bartlett, A. C., Hollot, C. V. e Lin, H. (1988). Root location of an entire polytope of polinomials: it suffices to check the edges, Mathematics of Controls, Signals and Systems 1: 61-71.

Bhattacharyya, S. P., Chapellat, H. e Keel, L. H. (1995). Robust Control: The Parametric Approach, Prentice-Hall, Upper Saddle River, NJ.

Chen, C. T. (1999). Linear System Theory and Design, 3rd. edition, Oxford University Press, Inc., New York.

Horst, R. e Pardalos, P. M. (1994). Hand-book of Global Optimization, Kluwer Academic Publishers, Dordrecht.

Ignizio, J. P. (1982). Linear Programming in Single and Multiple-Objective Systems, Prentice Hall, Englewood Cliffs, NJ.

Inuiguchi, M. е Kume, Y. (1991). Goal programming problems with interval coefficients and target intervals, European Journal of Operational Reasearch 52: 345 360 .

Jaulin, L., Kieffer, M., Didrit, O. e Walter, E. (2001). Applied Interval Analysis, Springer-Verlag, London. 
Keel, L. H. e Bhattacharyya, S. P. (1997a). A linear programming approach to controller design, Proceedings of the 36th IEEE Conference on Decision and Control, San Diego, CA, USA, pp. 2139-2148.

Keel, L. H. e Bhattacharyya, S. P. (1997b). Robust, fragile or optimal?, IEEE Transactions on Automatic Control 42: 1098-2005.

Keel, L. H. e Bhattacharyya, S. P. (1999). Robust stability and performance with fixed-order controllers, Automatica 35: 1717-1724.

Kelling, B. (1994). Geometric analysis of bounded solution sets of systems of linear interval equations, Zeitschrift für Angewandte Mathematik und Mechanik 74: 625628.

Lordelo, A. D. S. e Ferreira, P. A. V. (2002a). Linear controller design by goal programming, Proceedings of the IEEE International Symposium on Computer Aided Control System Design, Glasgow, Escócia, pp. 145150.

Lordelo, A. D. S. e Ferreira, P. A. V. (2002b). Projeto de controladores lineares via programação alvo, Anais do XIV Congresso Brasileiro de Automática, Natal, Brasil, pp. 2409-2414.

Lordelo, A. D. S. e Ferreira, P. A. V. (2004). Analysis and design of robust pole placement controllers using the interval diophantine equation, (em preparação) .

Lordelo, A. D. S., Juzzo, E. A. e Ferreira, P. A. V. (2004a). On the design of robust controllers using the interval diophantine equation, Proceedings of the IEEE International Symposium on Computer Aided Control System Design, Taipei, Taiwan, pp. 173-178.

Lordelo, A. D. S., Juzzo, E. A. e Ferreira, P. A. V. (2004b). Projeto de controladores robustos através da equação diofantina intervalar, Anais do XV Congresso Brasileiro de Automática, Gramado, Brasil.

Moore, R. E. (1979). Methods and Applications of Interval Analysis, SIAM, Philadelphia.

Oettli, W. (1965). On the solution of a linear system with inaccurate coefficients, SIAM Journal of Numerical Analysis 2: 115-118.

Oettli, W. e Prager, W. (1964). Compatibility of approximate solution of linear equations with given error bounds for coefficients and right-hand sides, Numer. Math. 6: 405409.

Prado, M. L. M., Lordelo, A. D. S. e Ferreira, P. A. V. (2004). An interval analysis approach for the design of robust state feedback controllers, (em preparação) .
Rohn, J. (1986). Inner solutions of linear interval equations, Lecture Notes in Computer Science. Springer-Verlag, Berlin-Heidelberg 212: 157-158.

Rohn, J. (1989). Systems of linear interval equations, Linear Algebra and Applications 126: 39-78.

Rotstein, H., Peña, R. S., Bandoni, J., Desages, A. e Romagnoli, J. (1991). Robust characteristic polynomial assignment, Automatica 27: 711-715.

Soh, Y. C., Evans, R. J., Petersen, I. e Betz, R. E. (1987). Robust pole assignment, Automatica 23: 601-610.

Yu, P. L. (1985). Multiple Criteria Decision MakingConcepts, Techniques and Extensions, Plenum Press, New York, NY. 